\title{
Simulation and Application of Power System Stabilizer on Power System Transient Stability
}

\author{
Ang $\mathrm{Li}^{*}$ \\ School of Electrical Engineering, Shaanxi University of Technology, Chaoyang Road, Hanzhong, Shaanxi, 723000, \\ China
}

\begin{abstract}
This paper introduces the working principle and the mathematical model of additional power system excitation control-Power System Stabilizer (PSS). Through established a typical single machine-infinite bus power system simulation model, we simulate the synchronous generator's transient operational characteristics following a severe disturbance. The simulation results show that the PSS can not only effectively increase the system damping, but also improve operational characteristics of the generator, considerably enhance power system dynamic and transient stability.
\end{abstract}

Keywords: Low frequency oscillation, MATLAB simulation, power system stabilizer, transient stability.

\section{INTRODUCTION}

The synchronous generator's excitation system plays an important role in advancing power system's reliability and stability. Enhancing the performance of excitation devices has been acknowledged as one of the most efficient and economic method to improve generator's stability. Conventional PID control based on classical control theory has been widely used, but PID control adjusted only by terminal voltage deviation can not provide satisfactory damping characteristics, especially for the control of large generator units, that the damping characteristics will produce spontaneous lowfrequency oscillation, threatening the power system stability [1]. The effective measures to solve the contradiction between voltage regulation accuracy and dynamic stability is the additional power system stabilizer (PSS), which can effectively enhance the damping of power system, thereby improve its static and dynamic stability.

\section{WORKING PRINCIPAL AND MATHEMATICAL MODEL OF THE PSS}

\subsection{Low-Frequency Oscillation Mechanisms}

Large synchronous generator excitation system is generally composed of two parts by the excitation power unit and the excitation control unit.

Be adjusted in accordance with the generator terminal voltage deviation, the excitation control unit, also known as automatic voltage regulator (AVR), is the main excitation controller. With the regulation accuracy of the AVR is getting higher and higher, the response of the excitation power is also growing fast. Fast excitation system can indeed improve the synchronization torque, thereby enhancing transient stability of the system in first oscillation cycle. However, the fast excitation system is generally negative feedback system with high magnification, which has little effect on the system oscillation damping after the first swing cycle, and sometimes even reduce system oscillation damping. If the system present negative damping properties, fast excitation system (especially high magnification) is to increase negative damping, which worsened the operation status of the system [2].

\subsection{Mathematical Model of the PSS}

PSS is an additional excitation control. Its essence is to provide an additional signal to increase damping torque of the generator excitation system. This control signal can provide positive damping effect, without affecting the voltage regulation function of the voltage regulator and the ability of maintaining the generator terminal voltage level, therefore, also known as additional excitation control.

For the purpose to increase the capability of preventing power system from low-frequency oscillation, PSS can use the generator rotor speed $(\Delta \omega)$, system frequency $(\Delta f)$, accelerating power $\left(P_{m}-P_{e}\right)$, electrical power $\left(\Delta P_{e}\right)$, and the combination of these signals as input signals [3].

For the output $V_{P S S}$ of PSS is a supplementary excitation control signal, with Phillips-Heffron model [4] shown as Fig. (1), we know the power deviation is:

$$
\Delta P_{e}=\frac{T_{e} K_{2} K_{4} s+K_{2} K_{4}+K_{2} K_{5} K_{e}}{T_{d 0}^{\prime} T_{e} s^{2}+\left(T_{d 0}^{\prime}+\frac{T_{e}}{K_{3}}\right) s+\left(\frac{1}{K_{3}}+K_{e} K_{6}\right)} \Delta \delta,
$$




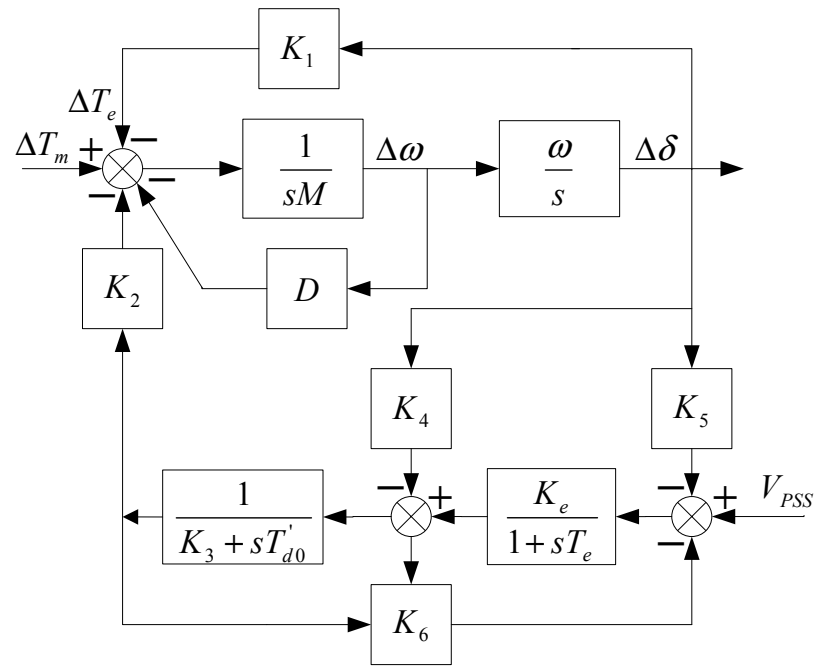

Fig. (1). Transfer function of single machine-infinite bus power system.

$$
\left\{\begin{array}{l}
K_{1}=\frac{\partial P_{e}}{\partial \delta}=\frac{X_{q}-X_{d}^{\prime}}{X_{d}^{\prime}+X_{e}} I_{q 0} \Delta E_{q}^{\prime}+\frac{U_{c} \cos \delta_{0}}{X_{q}+X_{e}} E_{Q 0} \\
K_{2}=\frac{\partial P_{e}}{\partial E_{q}^{\prime}}=\frac{X_{q}+X_{e}}{X_{d}^{\prime}+X_{e}} I_{q 0} \\
K_{3}=\frac{\partial E_{q}}{\partial E_{q}^{\prime}}=\frac{X_{d}^{\prime}+X_{e}}{X_{d}+X_{e}} \\
K_{4}=\frac{\partial E_{q}}{\partial \delta}=\frac{X_{d}-X_{d}^{\prime}}{X_{d}^{\prime}+X_{e}} U_{c} \sin \delta_{0} \\
K_{5}=\frac{\partial V_{t}}{\partial \delta}=\frac{U_{t d 0}}{U_{t 0}} \frac{X_{q}}{X_{q}+X_{e}} U_{c} \cos \delta_{0}-\frac{U_{t q 0}}{U_{t 0}} \frac{X_{d}^{\prime}}{X_{d}+X_{e}} U_{c} \sin \delta_{0} \\
K_{6}=\frac{\partial V_{t}}{\partial E_{q}^{\prime}}=\frac{U_{t q 0}}{U_{t 0}} \frac{X_{e}}{X_{d}^{\prime}+X_{e}}
\end{array}\right.
$$

Where $X_{e}$ is the link-reactance between the generator and the system, and $U_{c}$ is the terminal voltage of the infinite bus, others are parameters of the generator and operating variables of the system. Obviously, the values of $K_{1} \sim K_{6}$ reflect the generator's operating condition and the connection with the system (which $K_{3}$ has nothing to do with the operation condition), apparently operating conditions and grid parameters will affect the values of $K_{1} \sim K_{6}$.

If the armature reaction is neglected (i.e., $K_{4}=0$ ), we get the simple results:

$$
\Delta P_{e}=\frac{K_{2} K_{5} K_{e}}{T_{d 0}^{\prime} T_{e} s^{2}+\left(T_{d 0}^{\prime}+\frac{T_{e}}{K_{3}}\right) s+\left(\frac{1}{K_{3}}+K_{e} K_{6}\right)} \Delta \delta,
$$

Substituting $s=j \omega$ into (2) and then it can be separated into real and imaginary parts, we get:

$\Delta P_{e}=K_{a} \Delta \delta+D_{a} \Delta \omega$
Where: $K_{a}=\frac{K_{2} K_{5} K_{e}\left(1 / K_{3}+K_{e} K_{6}-\omega^{2} T_{d 0}^{\prime} T_{e}\right)}{\left(1 / K_{3}+K_{e} K_{6}-\omega^{2} T_{d 0}^{\prime} T_{e}\right)^{2}+\omega^{2}\left(T_{d 0}^{\prime}+T_{e} / K_{3}\right)^{2}}$

is synchronous torque coefficient, and $D_{a}=\frac{K_{2} K_{5} K_{e}\left(T_{d 0}^{\prime}+T_{e} / K_{3}\right) \omega_{0}}{\left(1 / K_{3}+K_{e} K_{6}-\omega^{2} T_{d 0}^{\prime} T_{e}\right)^{2}+\omega^{2}\left(T_{d 0}^{\prime}+T_{e} / K_{3}\right)^{2}}$ is damping torque coefficient.

Due to the parameters characteristics of non-salient pole generator $\left(X_{d}>X_{q}>X_{q}^{\prime}>X_{d}^{\prime}\right)$ and salient pole generator $\left(X_{d}=X_{q}>X_{q}^{\prime}>X_{d}^{\prime}\right)$, usually $K_{1}, K_{2}, K_{3}, K_{4}, K_{6}$ are positive. In heavy-duty, it is clear that with power angle increase, $K_{5}$ will be negative and $D_{a}<0$, thus the system differential equations characteristic root is positive, the system may be negative damping oscillation.

For conventional excitation system, because $K_{e} K_{6} \ll<\omega^{2} T_{d 0}^{\prime} T_{e}$, and $T_{d 0}^{\prime}$ is considerable (4 8s), then the damping coefficient can be approximately written as:

$$
\begin{aligned}
& D_{a}=\frac{K_{2} K_{5} K_{e}\left(T_{d 0}^{\prime}+T_{e} / K_{3}\right) \omega_{0}}{\left(1 / K_{3}-\omega^{2} T_{d 0}^{\prime} T_{e}\right)^{2}+\omega^{2}\left(T_{d 0}^{\prime}+T_{e} / K_{3}\right)^{2}} \\
& =\frac{K_{2} K_{5} K_{e}\left(T_{d 0}^{\prime}+T_{e} / K_{3}\right) \omega_{0}}{\left(1+\omega^{2} T_{e}^{2}\right)\left(\omega^{2} T_{d 0}^{\prime}{ }^{2}+1 / K_{3}^{2}\right)} \approx \frac{K_{2} K_{5} K_{e} \omega_{0}}{\omega^{2} T_{d 0}^{\prime}\left(1+\omega^{2} T_{e}^{2}\right)}
\end{aligned}
$$

Thus, the greater the value of $K_{e}$, the greater the negative damping, and the oscillation will be more serious. With the value of $T_{e}$ increases, the negative damping can be reduced, so that the oscillation weakened. However, the value of $T_{e}$ cannot be too big, otherwise the oscillation time will be extended, that is not conducive to the stability of the system.

For the value of $T_{e}\left(T_{e} \rightarrow 0\right)$ is very small in fast excitation system, so the negative damping is enhanced, resulting in a low-frequency oscillation. Thus PSS is generally installed in fast excitation system. When $K_{e}$ is large, the damping coefficient can be approximately written as:

$$
D_{a} \approx \frac{K_{2} K_{5} K_{e} T_{d 0}^{\prime} \omega_{0}}{\omega^{2}\left(T_{d 0}^{\prime}\right)^{2}+\left(K_{e} K_{6}\right)^{2}},
$$

It can be seen that when $K_{e}$ is greater than a certain value, the denominator increases faster than the molecule, the negative damping will decrease thus fast excitation system can be stable even if the gain of AVR very large.

From Fig. (1), the system electromagnetic torque can be written as:

$$
\begin{aligned}
& \Delta T_{e}=\left[K_{1}-\frac{K_{2} K_{e}}{\left(K_{3}+T_{d 0}^{\prime} s\right)\left(1+T_{e} s\right)+K_{e} K_{6}}\left(K_{5}+K_{4} \frac{1+T_{e} s}{K_{e}}\right)\right] \Delta \delta \\
& =\left[K_{1}-\frac{K_{2} K_{4}}{\left(K_{2}+T_{d 0}^{\prime} s\right)+\frac{K_{e} K_{6}}{1+T_{e} s}}-\frac{K_{2} K_{e} K_{5}}{\left(K_{3}+T_{d 0}^{\prime} s\right)\left(1+T_{e} s\right)+K_{e} K_{6}}\right] \Delta \delta
\end{aligned}
$$




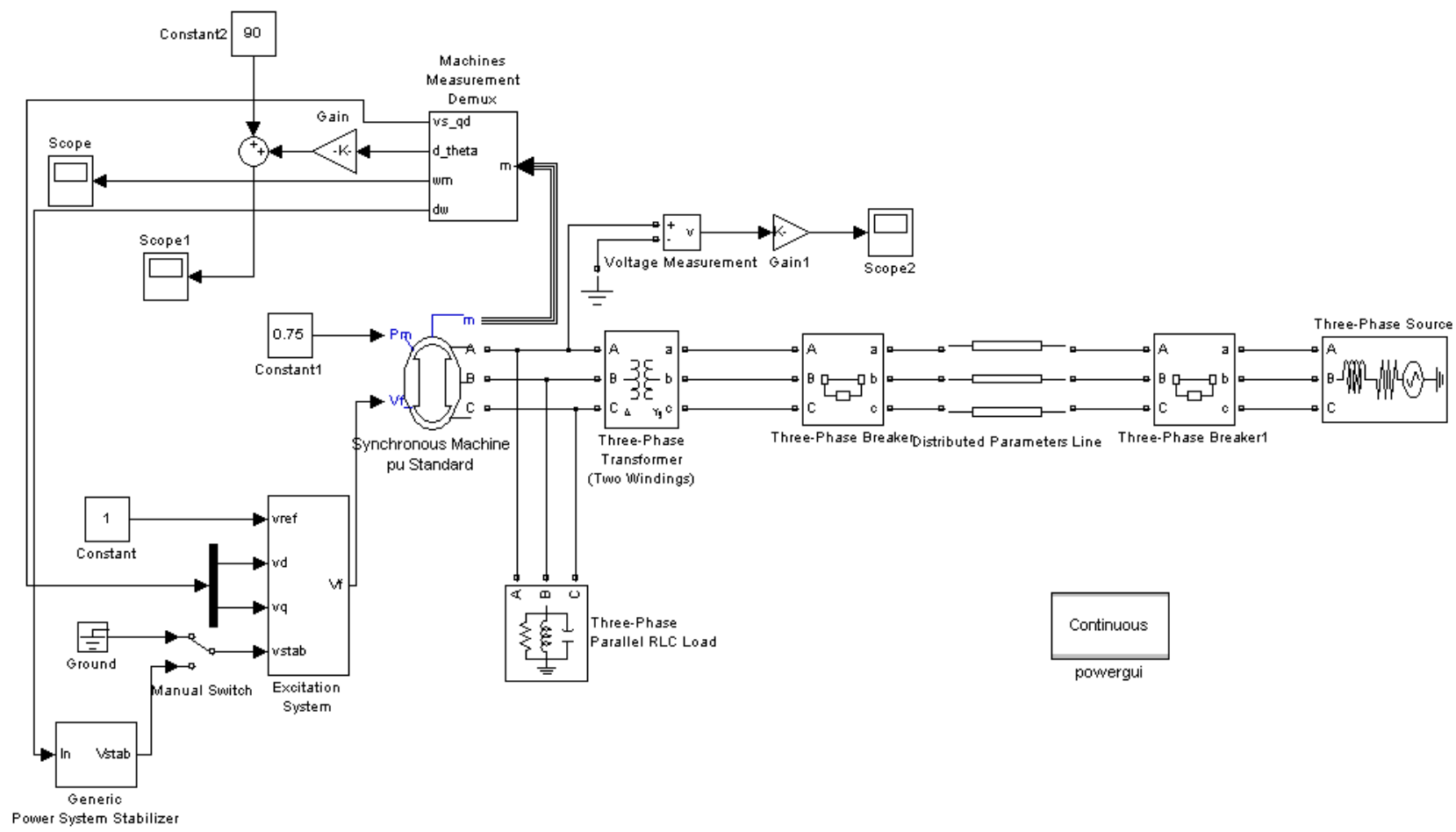

Fig. (2). Simulation model of the single machine-infinite bus power system.

Let $\Delta \delta$ be a sinusoidal perturbation, assume the angular velocity is $\Omega$, then $\Delta \omega=s \Delta \delta=j \Omega \Delta \delta$.

Taking $s=j \Omega$ into the above equation, we obtain:

$$
\begin{gathered}
\Delta T_{e}=\left[K_{1}-\frac{K_{2} K_{4}}{\left(K_{3}+j \Omega T_{d 0}^{\prime}\right)+\frac{K_{e} K_{6}}{1+j \Omega T_{e}}},\right. \\
\left.-\frac{K_{2} K_{e} K_{5}}{\left(K_{3}+j \Omega T_{d 0}^{\prime}\right)\left(1+j \Omega T_{e}\right)+K_{e} K_{6}}\right] \Delta \delta
\end{gathered}
$$

Taking $\Delta \omega$ as input signal, the transfer function of PSS is $G_{P S S}(s)=G_{P S S} \angle \Phi_{P S S}$, the additional damping torque is:

$$
\begin{aligned}
& \Delta T_{e}^{*}=\frac{K_{2} K_{E}}{\left(K_{3}+s T_{d 0}^{\prime}\right)\left(1+s T_{E}\right)+K_{E} K_{6}} G_{P S S}(s) \Delta \omega, \\
& =\frac{K_{2} K_{E}}{G_{3} \angle \Phi_{3}} G_{P S S} \angle \Phi_{P S S} \Delta \omega,
\end{aligned}
$$

If the leading phase $\Phi_{P S S}$ is equal to the denominator in phase $\Phi_{3}$, there are:

$\Delta T_{e}^{*}=\frac{K_{2} K_{E}}{G_{3}} G_{P S S} \Delta \omega$

It can be seen that the $\Delta T_{e}^{*}$ adds a component because of PSS, which the same phase with $\Delta \omega$, thereby generating positive damping. Generally with $K_{E}$ increases, $\Delta T_{e}^{*}$ becomes larger, that the PSS is best to install as additional ex- citation control signals in high magnification excitation system.

\section{TRANSIENT STABILITY SIMULATION OF THE PSS}

\subsection{Single Machine-Infinite Bus System Model}

Through established a simulation model of a typical single machine-infinite bus power system, as shown in Fig. (2), we simulate the synchronous generator transient operating characteristics following a severe disturbance.

The generator system consists of synchronous generator and excitation control system (AVR+PSS). Infinite system uses three-phase power supply module. In this case we assume that the mechanical power input to the generator is constant. And the solver algorithm adopts ode23tb. is:

If taking $\Delta \omega$ as input signal, the transfer function of PSS

$$
P(S)=20 \times \frac{2 s}{1+2 s} \times\left(\frac{1+0.05 s}{1+0.02 s}\right) \times\left(\frac{1+3 s}{1+5.4 s}\right),
$$

The output limits are $\pm 15 \%$.

\subsection{The Influence of PSS upon Transient Stability}

The main cause leading instability under great disturbance is the power difference (i.e. accelerating power) between mechanical power and electromagnetic power of the generator, thus reducing the power difference is first of all consider measures (such as fast fault clearing, exciter control, fast closing main valve etc.). On this basis, the influence 


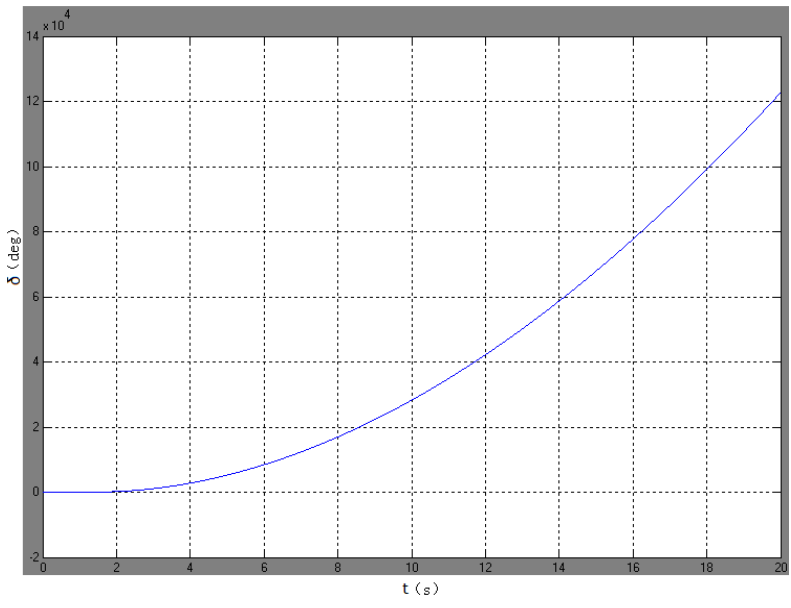

$\mathbf{a}$

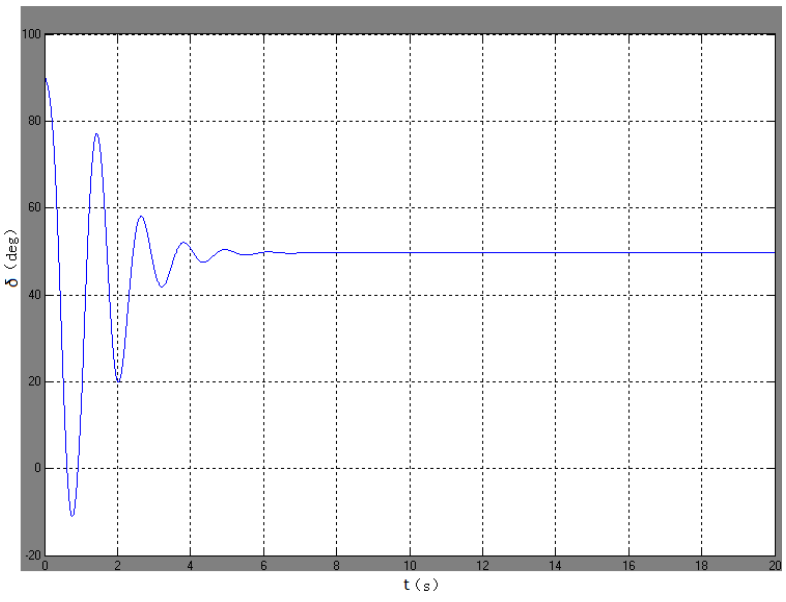

b

Fig. (3). Simulation curves of generator power angle: (a) without PSS, (b) with PSS.

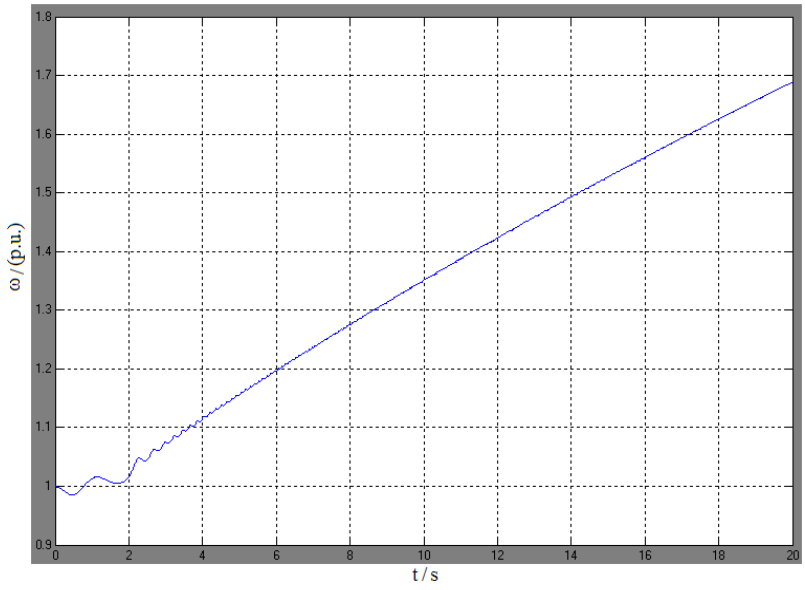

$\mathbf{a}$

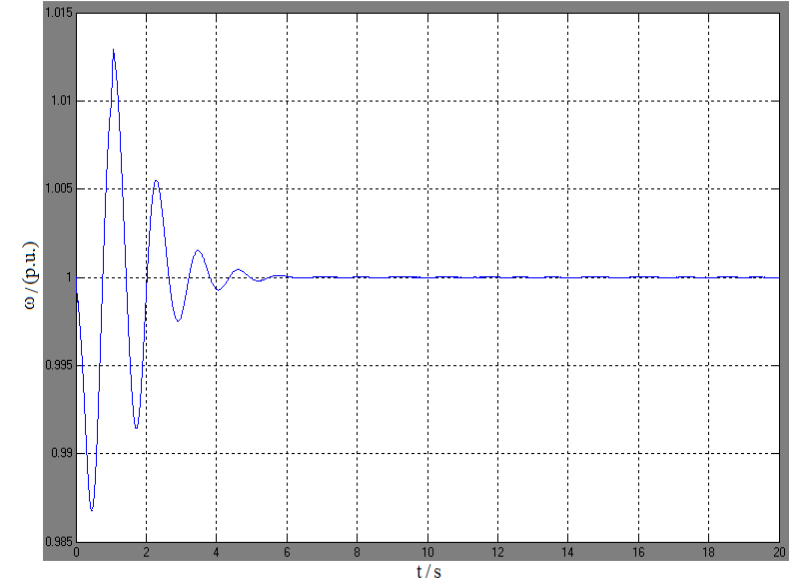

b

Fig. (4). Simulation curves of generator rotor speed: (a) without PSS, (b) with PSS.

of the PSS to power system transient stability has been studied.

The fault of three-phase short circuit occurs at $\mathrm{t}=0 \mathrm{~s}$ at the head of the transmission line, and the fault clears at $t=1.06 \mathrm{~s}$. Power angles and rotor speed curves of generator with or without PSS are respectively shown in Fig. (3) and Fig. (4).

For severe disturbance such as three-phase short-circuit, the research results show that when PSS is not equipped, despite applying the measures such as removal the fault rapidly, the system still lost its stability. Therefore, when PSS is applied, the damping of the system will be remarkably enhanced and the system stability will be improved. The PSS makes the generator to produce additional electromagnetic power, enhance the damping of the system, so the first angle oscillation back swing amplitude decreased, significantly improves the operating characteristics of generator.

\section{CONCLUSION}

This paper takes $\Delta \omega$ as the input signal to PSS as an additional excitation control, simulates the transient process of a single machine infinite-bus system in large disturbance, qualitative analysis the stability of the excitation control system. From the simulation results we can see that the PSS can provide sufficient positive damping, effectively overcome the negative damping of the AVR, and significantly improve the transient stability of power system. But PSS for the role of the degree of power system stability depends mainly on two aspects: one is the site where the PSS should be installed; the other is the parameters of PSS were optimized [5]. In complex multi-machine power system, the choice of the model and configuration is quite complicated and important. If the choice is undeserved, even make the low frequency oscillation more serious.

\section{CONFLICT OF INTEREST}

The author confirms that this article content has no conflict of interest.

\section{ACKNOWLEDGEMENTS}

The author gratefully acknowledge the financial supports from Hanzhong Municipal Bureau of Science and Technology Fund (No. 2013hzzx-61). 


\section{REFERENCES}

[1] W.B. Chu, J.M. Liu, J.Z. Liu, and L.X. Tan, “ Modeling and Simulation about Included New PSS of Excitation System", Electric Machines \& Control Application, vol. 12, pp. 6-9, 2009.

[2] X.F. Wang, W.L. Fang, and Z.C. Du, Modern Power System Analysis. ${ }^{\text {st }}$ ed. Science Press: Beijing, 2003, pp. 399-420. (in Chinese).

[3] H.L. Yang, T.Y. Zhang, and J.J. Guo, " Action of PSS on system stability and parameter stablishment in field regulator", North China Electric Power, vol. 12, pp. 12-14, 2005.
[4] M. Huang, and H.Y. Han, "The PSS parameters optimization based-on power system operating variables", Electric Power, vol. 5, pp. 19-22, 2007.

[5] F. Zhang, J.F. Li, W. Wang, H.P. Zhou, Z.H. Xiang, G.S. Ye, and Y.P. Zhou, "The simulation of PSS and SVC to stability of electric power transmission based on MATLAB”, Electric Switchgear, vol. 1, pp. 40-42, 2009

Received: November 20, 2014

Revised: January 07, 2015

Accepted: January 20, 2015

(C) Ang Li; Licensee Bentham Open.

This is an open access article licensed under the terms of the Creative Commons Attribution Non-Commercial License (http://creativecommons.org/licenses/by$\mathrm{nc} / 3.0 /$ ) which permits unrestricted, non-commercial use, distribution and reproduction in any medium, provided the work is properly cited. 